\title{
Editorial
}

\section{Welcome to Network: A New Open-Access Scientific Journal}

\author{
Alexey Vinel $\mathbb{B}$

Network (ISSN 2673-8732) provides full coverage of all topics of interest involved in the networking area. The purpose of this journal is to bring together researchers, engineers, and students from academia and industry to present novel ideas and solid research about the theoretical and practical aspects in the application domains of communications and networks. The primary focus of the journal is on the analysis, modeling, design, simulation, and implementation of networks. This journal will also serve to attract research concerning applying networking architectures and scenarios to emerging research topics such as Internet of Things (IoT), smart cities, and edge computing, among others.

We welcome researchers worldwide to contribute to Network in any emerging or consolidated topics of network including but not limited to the following:

- Physical layer techniques;

- Network protocols and architectures;

- Mobile and wireless networks;

- Network management;

- Network security;

- Applications and services.

Network aims to facilitate increased collaboration between scientists and engineers around the world to produce innovative ideas and cutting-edge technologies that will be submitted to Network. We expect to be of significance for any member of the scientific community enthusiastic in participating in this project.

We look forward to your participation in this exciting journal.

Conflicts of Interest: The author declares no conflict of interest.

\section{Short Biography of Author}

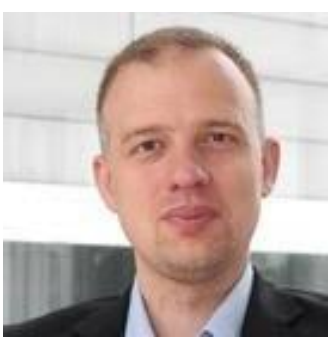

\begin{abstract}
Alexey Vinel received his PhD degrees from the Institute for Information Transmission Problems, Moscow, Russia, in 2007, and from the Tampere University of Technology, Tampere, Finland, in 2013. He has been a Professor with the School of Information Technology, Halmstad University, Halmstad, Sweden since 2015, and a Professor II with the Department of Electrical Engineering, Western Norway University of Applied Sciences, Bergen, Norway since 2018. His areas of interest include wireless communication, vehicular networking, and cooperative autonomous driving.
\end{abstract}

